OPEN ACCESS

Edited by:

Rekha Seshadri,

Department of Energy Joint Genome Institute, USA

Reviewed by:

Uli Stingl,

King Abdullah University of Science and Technology, Saudi Arabia

Jonathan Badger,

National Cancer Institute, USA

*Correspondence:

Francisco J. Silva

francisco.silva@uv.es

Specialty section:

This article was submitted to

Evolutionary and Genomic

Microbiology,

a section of the journal

Frontiers in Microbiology

Received: 07 September 2015

Accepted: 31 October 2015

Published: 13 November 2015

Citation:

Silva FJ and Santos-Garcia D (2015)

Slow and Fast Evolving Endosymbiont

Lineages: Positive Correlation between the Rates of Synonymous and Non-Synonymous Substitution.

Front. Microbiol. 6:1279.

doi: 10.3389/fmicb.2015.01279

\section{Slow and Fast Evolving Endosymbiont Lineages: Positive Correlation between the Rates of Synonymous and Non-Synonymous Substitution}

\author{
Francisco J. Silva ${ }^{1,2 *}$ and Diego Santos-Garcia ${ }^{3}$ \\ ${ }^{1}$ Institut Cavanilles de Biodiversitat i Biologia Evolutiva, Universitat de València, València, Spain, ${ }^{2}$ Unidad Mixta de \\ Investigación en Genómica y Salud, Fundación para el Fomento de la Investigación Sanitaria y Biomédica de la Comunitat \\ Valenciana, Salud Pública/Institut Cavanilles, Universitat de València, València, Spain, ${ }^{3}$ Department of Entomology, Hebrew \\ University of Jerusalem, Rehovot, Israel
}

The availability of complete genome sequences of bacterial endosymbionts with strict vertical transmission to the host progeny opens the possibility to estimate molecular evolutionary rates in different lineages and understand the main biological mechanisms influencing these rates. We have compared the rates of evolution for non-synonymous and synonymous substitutions in nine bacterial endosymbiont lineages, belonging to four clades (Baumannia, Blochmannia, Portiera, and Sulcia). The main results are the observation of a positive correlation between both rates with differences among lineages of up to three orders of magnitude and that the substitution rates decrease over long endosymbioses. To explain these results we propose three mechanisms. The first, variations in the efficiencies of DNA replication and DNA repair systems, is unable to explain most of the observed differences. The second, variations in the generation time among bacterial lineages, would be based on the accumulation of fewer DNA replication errors per unit time in organisms with longer generation times. The third, a potential control of the endosymbiont DNA replication and repair systems through the transfer of nuclear-encoded proteins, could explain the lower rates in long-term obligate endosymbionts. Because the preservation of the genomic integrity of the harbored obligate endosymbiont would be advantageous for the insect host, biological mechanisms producing a general reduction in the rates of nucleotide substitution per unit of time would be a target for natural selection.

\footnotetext{
Keywords: endosymbiosis, evolutionary rate, nucleotide substitution, generation time, DNA replication, DNA repair
}

\section{INTRODUCTION}

The main problem to estimate and compare the rates of sequence evolution among bacterial lineages is the difficulty to obtain the times of divergence of the compared species and strains. Although for culturable bacteria, in very short periods of time, an experimental approach in laboratory may be applied (Lenski et al., 2003), the estimations of the rates of nucleotide substitution over long periods of time are intractable. However, there is one exception: hostassociated bacteria. Because these organisms coevolve with eukaryotic lineages, the times of divergence of the hosts may be estimated with divergence dating methods using calibration points 
based on fossil records. Once estimated, host divergence times may be used to obtain the rates of nucleotide substitution in the endosymbionts, provided that transmission from host to offspring is strictly vertical. In insects, many endosymbiont lineages with strict vertical transmission for millions of years have been described (Baumann, 2005; Moran et al., 2008; Moya et al., 2008). They are ideal subjects to be analyzed although, until now, only some studies using rough estimations of the times of divergence among insect taxa have been performed. The use of methods for temporal calibration of molecular phylogenies permits the estimation of the rates of molecular evolution within any endosymbiotic clade with strictly vertical transmission.

\section{RATES OF GENE SEQUENCE EVOLUTION IN BACTERIAL ENDOSYMBIONTS}

The first comparisons of the rates of gene sequence evolution between endosymbionts and free living microorganisms were mainly focused on rRNA genes. It was observed through relative rate tests the significant acceleration of the 16S rRNA genes of the primary endosymbionts of aphids (Buchnera aphidicola), mealybugs, whiteflies, and tse-tse flies in comparison with close free-living relatives (Moran, 1996). This kind of fast evolution was later reported for sulfur-oxidizing bacteria (Peek et al., 1998), fungi (Lutzoni and Pagel, 1997), or fungi and bacteria (Woolfit and Bromham, 2003). These comparative analyses were extended to a few coding genes. For example, the number of nonsynonymous substitutions per site $(\mathrm{dN})$ was higher in several Buchnera genes than in their corresponding orthologs in enterics (Moran, 1996; Clark et al., 1999). However, the differences in the number of synonymous substitutions per site (dS) between the endosymbiont (Buchnera) and the free-living relative were small (Clark et al., 1999).

Later, the comparative analyses were extended to different endosymbiont lineages, among then and with their free-living relatives, and the estimated dS were weighted by estimations of the times of divergence. In this case, large differences were observed for the $\mathrm{dS} / \mathrm{t}$ (dS/time) of the genes gidA and groEL with estimations of around $1 \times 10^{-7}, 1 \times 10^{-8}$ and $1 \times 10^{-9}$ synonymous substitutions per site per year for "Candidatus Blochmannia" (endosymbiont of carpenter ants, hereafter Blochmannia), Buchnera and free-living enterics, respectively (Degnan et al., 2004). Rates of evolution for nonfunctional DNA sequences were also estimated for Buchnera and Blochmannia at $4.3 \times 10^{-9}$ and $1.5 \times 10^{-8}$, respectively (Gomez-Valero et al., 2007, 2008).

Although the analysis of a reduced set of genes may give clues about the rates of molecular evolution, the availability of the complete endosymbiont genomes may contribute to get a whole view of the rates of evolution in each symbiont lineage, not affected by the random or selective processes of each gene.

Recently, two analyses involving the estimation of $\mathrm{dN}$ and $\mathrm{dS}$ (or $\mathrm{dN} / \mathrm{t}$ and $\mathrm{dS} / \mathrm{t}$ ) at genomic scale and the comparison among different endosymbiont lineages have been performed (Bennett et al., 2014; Santos-Garcia et al., 2015). In the first study, two obligate endosymbionts ("Candidatus Sulcia muelleri” and "Candidatus Baumannia cicadellinicola," hereafter Sulcia and Baumannia, respectively) of two sharpshooters species (Hemiptera:Cicadellidae:Cicadellinae) of the tribes Proconiini (Homalodisca vitripennis) and Cicadellini (Graphocephala atropunctata) were analyzed. For both symbionts, the start of the symbiosis took place before the divergence of both insect lineages, but while Sulcia has a long-term coevolutionary history with Auchenorrhyncha, the relation of Baumannia with sharpshooters is much more recent, because it replaced another ancestral symbiont in the subfamily Cicadellinae, probably as a consequence of a diet change from phloem to xylem (Bennett and Moran, 2013). Because the same divergence time may be applied to the two Baumannia strains and the two Sulcia strains, $\mathrm{dN}$ and $\mathrm{dS}$ were compared in spite of the absence of a concrete time of divergence. The average gene $\mathrm{dN}$ was 28 -fold higher in Baumannia than in Sulcia, while the differences in average gene dS were even higher (89-fold) (Bennett et al., 2014). This clearly differentiates Baumannia and Sulcia as fast and slow evolving endosymbionts.

In the second study, the rates of $\mathrm{dN} / \mathrm{t}$ and $\mathrm{dS} / \mathrm{t}$ were estimated in four lineages of "Candidatus Portiera aleyrodidarum" (hereafter Portiera), the endosymbiont of whiteflies (SantosGarcia et al., 2015). The divergence times of the lineages were estimated with the program BEAST (Bouckaert et al., 2014), sequence datasets from the endosymbionts and mitochondria and calibrators for the nodes of divergence of the two whitefly subfamilies (125-135 My) and of aphids and whiteflies (250$278 \mathrm{My}$ ). The results showed significant differences in the rate of evolution of the lineages, and an interesting correlation between $\mathrm{dN} / \mathrm{t}$ and $\mathrm{dS} / \mathrm{t}$, with Portiera from Bemisia tabaci evolving for both rates fivefold higher than the slow-evolving Portiera from Aleurodicus floccissimus. High $\mathrm{dN}$ and $\mathrm{dS}$ values in Portiera from B. tabaci had been previously reported when compared to Portiera from Trialeurodes vaporariorum (Sloan and Moran, 2013).

With the aim to compare at genomic scale the rates of nucleotide substitution in several endosymbiont lineages, we selected two long-term and two relatively recent endosymbiont clades. Sulcia and Portiera were the long-term clades. The infection of Sulcia took place 260-280 My ago (Moran et al., 2005), while in Portiera, the original infection probably took place in the ancestors of psyllids and whiteflies (>250 My ago), leading with time to the endosymbiotic genera "Candidatus Carsonella" and Portiera, respectively (Santos-Garcia et al., 2014). Baumannia, whose infection took place at least 50 My ago (Koga and Moran, 2014) and Blochmannia, whose infection of the ant tribe Camponotini took placed around 40 Mya (Williams and Wernegreen, 2015) were the short-term clades.

Only the $\mathrm{dN} / \mathrm{t}$ and $\mathrm{dS} / \mathrm{t}$ values from four Portiera lineages were directly obtained from a previous report (Santos-Garcia et al., 2015), while values for the remaining lineages were estimated for this work. Baumannia (BAU) and Sulcia (SUL) dN and dS gene estimations were obtained from the Supplementary Material of a previous publication (Bennett et al., 2014). In Sulcia, only the 
gene set retained for average estimations by Bennett et al. (2014), excluding those with zero $\mathrm{dN}$ or $\mathrm{dS}$ values and in Baumannia, the unsaturated genes. Because $\mathrm{dN}$ and $\mathrm{dS}$ were pairwise estimations, we obtained the $d N / t$ and $d S / t$ values corresponding to the substitutions in the two analyzed branches (Figure 1A) using twice the time of divergence of the lineages. We used a time of divergence between $H$. vitripennis and G. atropunctata of 66.2 My, an average of the estimations of $69 \mathrm{My}$ and 63.4 My obtained for their Sulcia and Baumannia endosymbionts (Szklarzewicz et al., 2015), because the value should be the same if both symbionts have strict vertical transmission.

Finally, we estimated the $\mathrm{dN}$ and $\mathrm{dS}$ gene values from the gene sequences reported in the genomes of Blochmannia floridanus, endosymbiont of Camponotus floridanus (Gil et al., 2003), Blochmannia pennsylvanicus, endosymbiont of Camponotus pennsylvanicus (Degnan et al., 2005) and the more distant relative, Blochmannia obliquus endosymbiont of Colobopsis obliquus (Williams and Wernegreen, 2015). To obtain these values, genomes of these species and Baummania as outgroup were obtained and their proteins classified in orthologous groups with OrthoMCL (Supplementary Material, Li et al., 2003). Then, codon alignments were obtained and codeML (Yang, 2007) was used to obtain the $\mathrm{dN}$ and $\mathrm{dS}$ values as previously described (Santos-Garcia et al., 2015). Only those genes with no zero $\mathrm{dN}$ or $\mathrm{dS}$ values and $\mathrm{dS}$ values less than four were selected for plotting and other analyses (the data files and the R markdown file can be found in Supplementary material). Three lineages were considered, the one leading to B. obliquus (BOB), whose period of evolution was estimated at 32.6 My (Degnan et al., 2004) and the two lineages leading to B. floridanus (BFL) and B pennsylvanicus (BPN), with a period of evolution of 18 My each (Degnan et al., 2004).

Gene $\mathrm{dN} / \mathrm{t}$ and $\mathrm{dS} / \mathrm{t}$ values in the nine lineages (Figure 1A) were plotted (Figure 1B). A significant positive correlation $\left(r^{2}=0.54\right)$ ( $p$-value $\left.<0.001\right)$ was observed under the linear regression model When the average values for $\mathrm{dN} / \mathrm{t}$ and $\mathrm{dS} / \mathrm{t}$ in each lineage were plotted (Figure 1C), a positive correlation $\left(r^{2}=0.98\right)$ was more clearly observed. Sulcia was the slowest evolving lineage followed by three out of the four Portiera lineages. Portiera from B. tabaci and Baumannia evolved with intermediate values. Finally, the rates of evolution of the three Blochmannia lineages were very high with the values of B. floridanus around 140 -fold and 600 -fold higher than Sulcia for $\mathrm{dN} / \mathrm{t}$ and $\mathrm{dS} / \mathrm{t}$, respectively. On the contrary, the average $\mathrm{dN} / \mathrm{dS}$ ratios were similar in most of the lineages, except in Sulcia which showed a value of around 0.30 (Figure 1D).

Although only a limited number of lineages have been analyzed, the main general conclusion that we can extract is that the evolution of the gene sequences in bacterial endosymbionts are triggered by mechanisms affecting both substitution rates. The second conclusion is that the long-term endosymbiotic clades (Sulcia and Portiera) evolve more slowly than the recent ones (Baumannia and Blochmannia). The exception is one of the lineages of Portiera, but it may be explained by the genome instability associated with this lineage (Sloan and Moran, 2012, 2013).

\section{EVOLUTIONARY PROCESSES EXPLAINING THE CORRELATION BETWEEN dN/t AND dS/t IN BACTERIAL ENDOSYMBIONTS}

The selection of four bacterial endosymbiotic clades for the analysis of the rates of coding gene evolution at genomic scale shows very large differences, in the range of three orders of magnitude between the slowest and the fastest evolving lineages. To explain this observation, we need to understand how endosymbiotic lifestyle may alter fundamental evolutionary processes such as mutation, selection, genetic drift, and recombination, and the profound consequences on the rates of nucleotide substitution (Wernegreen, 2015).

The heterogeneity of the gene $\mathrm{dN} / \mathrm{dS}$ ratios within a specific lineage may be conducted basically by natural selection. Positive selection may favor non-synonymous changes in some positions as an adaptation to changes in the intracellular environment, or purifying selection may remove a larger proportion of non-synonymous changes in some genes than in others. But, neither of these conditions affect both synonymous and nonsynonymous rates (Figure 1C). To explain this plot we need to consider mechanisms that alter simultaneously the rates of synonymous and non-synonymous substitutions. Both rates will not be the same because natural selection will favor the removal of most of the non-synonymous substitutions avoiding their transfer to the next generation but, in theory, it will have almost no effect over the synonymous ones, by its neutrality for most of the changes. However, natural selection may affect both rates simultaneously, if its action consists of selecting general mechanisms that either increase or decrease the mutation at any position of the DNA sequence. In this regard, we might predict that mechanisms lowering mutation rates would be favored in primary endosymbionts (Wernegreen, 2015).

Which are, thus, the mechanisms that can explain the differences of three orders of magnitude in the rates of the four analyzed symbiont clades? We propose three mechanisms, with the understanding that they are not mutually exclusive.

The first possible mechanism we considered is the efficiency of DNA replication and repair systems. The lower the efficiency, the higher the rates of mutation and substitution. The overall efficiency of these two systems involves many components. Not only is it difficult to analyze these components, but to determine how they are combined to generate an overall efficiency. Many mechanisms for DNA repair have been described in living beings including direct reversal, base excision repair, nucleotide excision repair and recombination (Sancar et al., 2004). Their efficiencies depend on many factors, such as, for example, the variety, concentration, error rate and activity of DNA replication and repair enzymes. The number of genes encoding proteins involved in DNA replication and DNA repair is one of these components (Table 1), although the observation of a lower number of genes in this category does not mean automatically a high substitution rate since it may be compensated by better performances of other components. Only within one clade, 

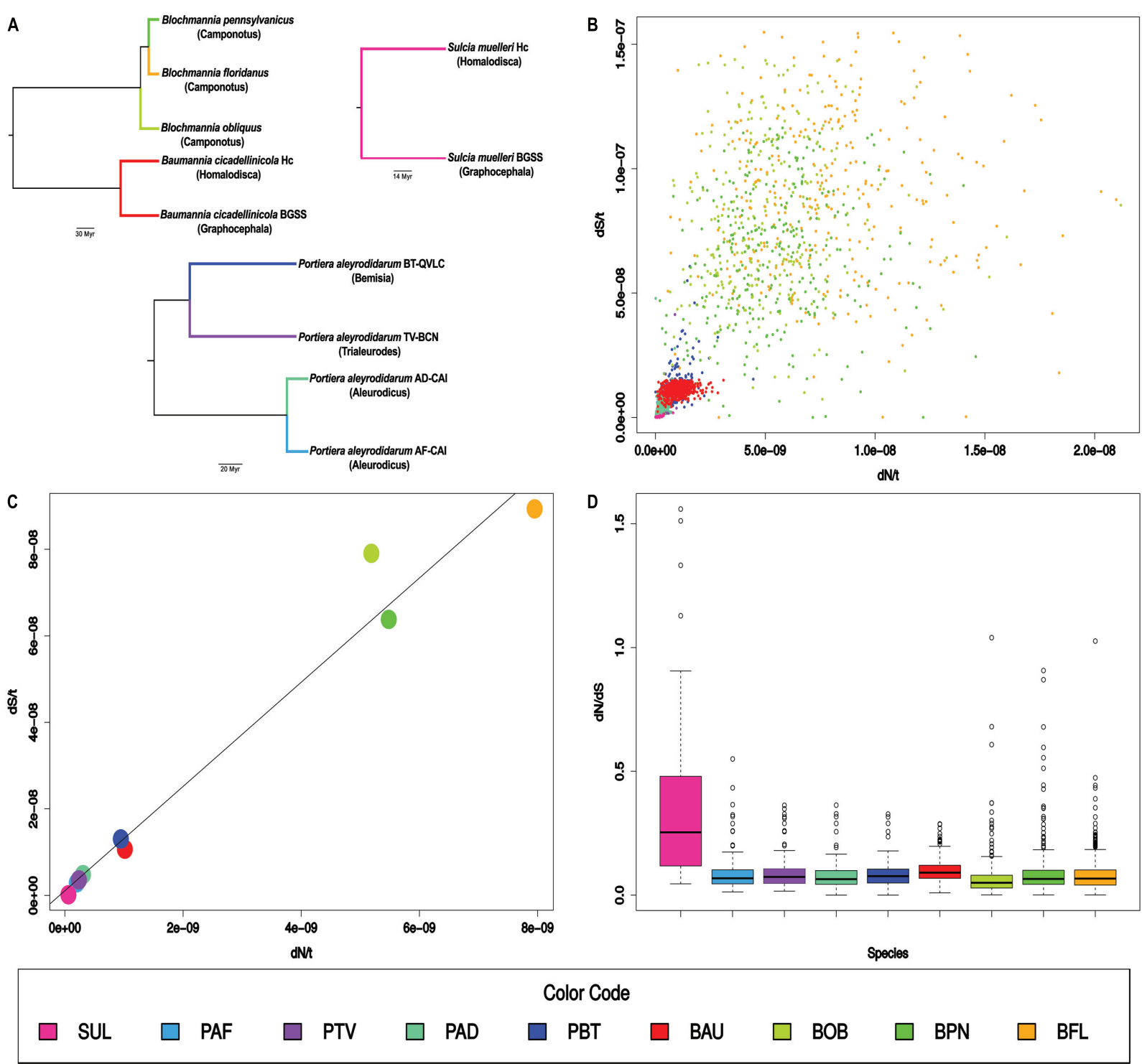

FIGURE 1 | Sequence evolution in bacterial endosymbiont lineages. (A) Tree topologies for the nine analyzed lineages (host genus between parentheses) were based on concatenated protein alignments (Bennett et al., 2014; Santos-Garcia et al., 2015; Bennett and Moran, 2013; Williams and Wernegreen, 2015). Branch colors mark the lineages. Notice that the analyses of Sulcia and Baumannia were not performed in a single branch. (B) Scatter plot of dN/t vs. dS/t. Each point is a gene. (C) Scatter plot of dN/t vs. dS/t. Each point is the average gene value for each lineage. A regression line is displayed. (D) Box plot for the dN/dS values of the genes in each lineage. Color codes and abbreviations are shown at the end. They were ordered according to dN/t from smallest to largest. Abbreviations: SUL, Sulcia; PAF, Portiera A. floccissimus; PTV, Portiera T. vaporariorum; PAD, Portiera A. dispersus; PBT, Portiera B. tabaci; BAU, Baumannia; BOB, B. obliquus; BPN, B. pennsylvanicus; and BFL, B. floridanus.

TABLE 1 | Number of genes in the DNA replication and repair category and rates of nucleotide substitution (average values).

\begin{tabular}{lccccccccc}
\hline & SUL & PAF & PTV & PAD & PBT & BAU & BOB & BPN & BFL \\
\hline Gene number & 6 & 13 & 13 & 13 & 5 & 26 & 18 & 18 \\
$\mathrm{dS} / \mathrm{t}$ & $1.5 \times 10^{-10}$ & $2.9 \times 10^{-09}$ & $3.6 \times 10^{-09}$ & $4.8 \times 10^{-09}$ & $1.3 \times 10^{-08}$ & $1.1 \times 10^{-08}$ & $7.9 \times 10^{-08}$ & $6.4 \times 10^{-08}$ & $8.9 \times 10^{-08}$ \\
$\mathrm{dN} / \mathrm{t}$ & $5.8 \times 10^{-11}$ & $2.0 \times 10^{-10}$ & $2.4 \times 10^{-10}$ & $3.1 \times 10^{-10}$ & $9.5 \times 10^{-10}$ & $1.0 \times 10^{-09}$ & $5.2 \times 10^{-09}$ & $5.6 \times 10^{-09}$ & $7.9 \times 10^{-09}$
\end{tabular}

See abbreviations in Figure 1.

we can observe some changes that may be associated with an increase of the mutation rate based on the loss of DNA repair and replication enzymes. This is the case of Portiera from B. tabaci B and Q biotypes (Santos-Garcia et al., 2012; Sloan and Moran, 2012) which has lost a large number of genes for DNA replication and repair comparing with the last 
common ancestor of Portiera strains (Santos-Garcia et al., 2015). However, when we compared the repertoires for these functional categories among the nine analyzed genomes, we did not find a general relationship between rates of substitution and number of genes in these categories and, in fact, the genera with the largest number of genes in these categories (Baumannia and Blochmannia), are those displaying the largest substitution rates (Table 1).

From the first theoretical studies, generation time was considered as one of the main factors contributing to explain the variability of the rates of molecular evolution among lineages. The generation time hypothesis was based on the idea that species with shorter generation times would have larger rates of mutations per year considering that larger numbers of DNA replications per unit of time generate larger numbers of mutations. Initially, the effect of generation time in vertebrates was only clearly observed in synonymous rates (Ohta, 1993). The difficulties to detect this effect in nonsynonymous rates were explained by the fact that species with larger generation times also tend to have smaller effective population sizes and both factors are compensated. Effects of generation time on the molecular evolutionary rates have also been described in other taxonomic groups such as invertebrates (Thomas et al., 2010) and, in this case, the effect was evident for both synonymous and non-synonymous rates.

In spite of the difficulties of study the effect of generation time in bacteria, it has been recently reported that, in Firmicutes, the DNA mutation and the protein evolutionary rates are smaller in spore-forming lineages than in non-spore-forming lineages (Weller and $\mathrm{Wu}, 2015$ ). Because, it is expected that the generation time is longer in spore-forming lineages, the authors indicate that their results support the effect of generation time also in bacteria.

In our analysis with endosymbionts, generation time may explain the observed differences, although no information over the number of DNA replications per unit of time may be easily estimated. However, the differences between B. floridanus and B. pennsylvanicus could be explained by the behavior of their hosts (Camponotus floridanus and Camponotus pennsylvanicus) with the former displaying year-round activity and the latter winter dormancy (Degnan et al., 2005). These host traits would probably affect to the number of bacterial generations and the rates of substitution per unit of time.

If the large differences among Sulcia and Portiera by one way and Baumannia and Blochmannia by the other are related to generation time, we cannot discard that it was natural selection, which selected that the former replicate their genomes with slower rates per unit of time than the latter. By this way, the degenerative effects associated with intracellular life will be weakened avoiding its replacement by a novel and more efficient endosymbiont (Santos-Garcia et al., 2015).

The third proposed mechanism is based on the recent demonstration that an insect protein was transferred to an endosymbiont, in the aphid endosymbiont B. aphidicola (Nakabachi et al., 2014) and the suggestion that the lack of a specific aminoacyl-tRNA synthetase in the endosymbiont "Candidatus Evansia muelleri" may be compensated by the import of the insect nuclear-encoded protein (SantosGarcia et al., 2014). After, a long-co-evolutionary relation, some insects would have taken power on their long-term endosymbionts, and to avoid their destruction, a mechanism of targeting some proteins inside the endosymbiont cell would have been developed. Some of the targeted proteins may be DNA repair enzymes, which would be able to remove many of the new mutations generated in the endosymbiont genome. Such a kind of mechanism for general decrease of the rates of mutation would have been selected and would explain why some endosymbiont lineages evolve with average rates for synonymous changes as small as $1 \times 10^{-10}$.

\section{CONCLUSION}

The huge variation among the rates of synonymous substitution among lineages of endosymbiotic bacteria cannot be explained by differences in the genetic arsenal required to avoid the production of mutations. The fact that this variation also affects to the non-synonymous rates requires that one or several general mechanisms decreasing the number of substitution by unit of time were involved. The generation time effect could explain this variability but, it is not necessary that the hosts display differences in their generation times. A slow rate of DNA replication and cell division in the endosymbionts will be sufficient to reduce the number of mutations per unit of time. The idea that the hosts may take the control of the endosymbiont rate of DNA replication or decrease the number of mutations by the transport of nuclear-encoded proteins within the endosymbiotic bacterial cell should be explored.

\section{AUTHOR CONTRIBUTIONS}

FS conceived research. FS and DS-G designed the analyses. DS-G performed the experiments. FS wrote the manuscript with inputs from DS-G. Both authors read and approved the final manuscript.

\section{FUNDING}

This work was supported by grants BFU2012-39816-C02-01 (cofinanced by FEDER funds and Ministerio de Economía y Competitividad, Spain) and PROMETEOII/2014/065 (Conselleria d'Educació, Generalitat Valenciana, Spain).

\section{SUPPLEMENTARY MATERIAL}

The Supplementary Material for this article can be found online at: http://journal.frontiersin.org/article/10.3389/fmicb. 2015.01279 


\section{REFERENCES}

Baumann, P. (2005). Biology of bacteriocyte-associated endosymbionts of plant sap-sucking insects. Annu. Rev. Microbiol. 59, 155-189. doi: 10.1146/annurev.micro.59.030804.121041

Bennett, G. M., McCutcheon, J. P., MacDonald, B. R., Romanovicz, D., and Moran, N. A. (2014). Differential genome evolution between companion symbionts in an insect-bacterial symbiosis. MBio 5, e1697-14. doi: 10.1128/mBio.01697-14

Bennett, G. M., and Moran, N. A. (2013). Small, smaller, smallest: the origins and evolution of ancient dual symbioses in a Phloem-feeding insect. Genome Biol. Evol. 5, 1675-1688. doi: 10.1093/gbe/evt118

Bouckaert, R., Heled, J., Kuhnert, D., Vaughan, T., Wu, C. H., Xie, D., et al. (2014). BEAST 2: a software platform for Bayesian evolutionary analysis. PLoS Comput. Biol. 10:e1003537. doi: 10.1371/journal.pcbi.10 03537

Clark, M. A., Moran, N. A., and Baumann, P. (1999). Sequence evolution in bacterial endosymbionts having extreme base compositions. Mol. Biol. Evol. 16, 1586-1598. doi: 10.1093/oxfordjournals.molbev.a026071

Degnan, P. H., Lazarus, A. B., Brock, C. D., and Wernegreen, J. J. (2004). Hostsymbiont stability and fast evolutionary rates in an ant-bacterium association: cospeciation of Camponotus species and their endosymbionts, Candidatus Blochmannia. Syst. Biol. 53, 95-110. doi: 10.1080/10635150490264842

Degnan, P. H., Lazarus, A. B., and Wernegreen, J. J. (2005). Genome sequence of Blochmannia pennsylvanicus indicates parallel evolutionary trends among bacterial mutualists of insects. Genome Res. 15, 1023-1033. doi: 10.1101/gr.3771305

Gil, R., Silva, F. J., Zientz, E., Delmotte, F., Gonzalez-Candelas, F., Latorre, A., et al. (2003). The genome sequence of Blochmannia floridanus: comparative analysis of reduced genomes. Proc. Natl. Acad. Sci. U.S.A. 100, 9388-9393. doi: $10.1073 /$ pnas. 1533499100

Gomez-Valero, L., Latorre, A., Gil, R., Gadau, J., Feldhaar, H., and Silva, F. J. (2008). Patterns and rates of nucleotide substitution, insertion and deletion in the endosymbiont of ants Blochmannia floridanus. Mol. Ecol. 17, 4382-4392. doi: 10.1111/j.1365-294X.2008.03912.x

Gomez-Valero, L., Silva, F. J., Simon, J. C., and Latorre, A. (2007). Genome reduction of the aphid endosymbiont Buchnera aphidicola in a recent evolutionary time scale. Gene 389, 87-95. doi: 10.1016/j.gene.2006.10.001

Koga, R., and Moran, N. A. (2014). Swapping symbionts in spittlebugs: evolutionary replacement of a reduced genome symbiont. ISME J. 8, 1237-1246. doi: 10.1038/ismej.2013.235

Lenski, R. E., Winkworth, C. L., and Riley, M. A. (2003). Rates of DNA sequence evolution in experimental populations of Escherichia coli during 20,000 generations. J. Mol. Evol. 56, 498-508. doi: 10.1007/s00239-002-2 423-0

Li, L., Stoeckert, C. J. Jr., and Roos, D. S. (2003). OrthoMCL: identification of ortholog groups for eukaryotic genomes. Genome Res. 13, 2178-2189. doi: $10.1101 /$ gr.1224503

Lutzoni, F., and Pagel, M. (1997). Accelerated evolution as a consequence of transitions to mutualism. Proc. Natl. Acad. Sci. U.S.A. 94, 11422-11427. doi: 10.1073/pnas.94.21.11422

Moran, N. A. (1996). Accelerated evolution and Muller's rachet in endosymbiotic bacteria. Proc. Natl. Acad. Sci. U.S.A. 93, 2873-2878. doi: 10.1073/pnas.93.7.2873

Moran, N. A., McCutcheon, J. P., and Nakabachi, A. (2008). Genomics and evolution of heritable bacterial symbionts. Annu. Rev. Genet. 42, 165-190. doi: 10.1146/annurev.genet.41.110306.130119

Moran, N. A., Tran, P., and Gerardo, N. M. (2005). Symbiosis and insect diversification: an ancient symbiont of sap-feeding insects from the bacterial phylum Bacteroidetes. Appl. Environ. Microbiol. 71, 8802-8810. doi: 10.1128/AEM.71.12.8802-8810.2005

Moya, A., Pereto, J., Gil, R., and Latorre, A. (2008). Learning how to live together: genomic insights into prokaryote-animal symbioses. Nat. Rev. Genet. 9, 218229. doi: $10.1038 / \mathrm{nrg} 2319$
Nakabachi, A., Ishida, K., Hongoh, Y., Ohkuma, M., and Miyagishima, S. Y. (2014). Aphid gene of bacterial origin encodes a protein transported to an obligate endosymbiont. Curr. Biol. 24, R640-R641. doi: 10.1016/j.cub.2014.0 6.038

Ohta, T. (1993). An examination of the generation-time effect on molecular evolution. Proc. Natl. Acad. Sci. U.S.A 90, 10676-10680. doi: 10.1073/pnas.90.22.10676

Peek, A. S., Vrijenhoek, R. C., and Gaut, B. S. (1998). Accelerated evolutionary rate in sulfur-oxidizing endosymbiotic bacteria associated with the mode of symbiont transmission. Mol. Biol. Evol. 15, 1514-1523. doi: 10.1093/oxfordjournals.molbev.a025879

Sancar, A., Lindsey-Boltz, L. A., Unsal-Kacmaz, K., and Linn, S. (2004). Molecular mechanisms of mammalian DNA repair and the DNA damage checkpoints. Annu. Rev. Biochem. 73, 39-85. doi: 10.1146/annurev.biochem.73.011303.073723

Santos-Garcia, D., Farnier, P. A., Beitia, F., Zchori-Fein, E., Vavre, F., Mouton, L., et al. (2012). Complete genome sequence of "Candidatus Portiera aleyrodidarum" BT-QVLC, an obligate symbiont that supplies amino acids and carotenoids to Bemisia tabaci. J. Bacteriol. 194, 6654-6655. doi: 10.1128/JB.01793-12

Santos-Garcia, D., Latorre, A., Moya, A., Gibbs, G., Hartung, V., Dettner, K., et al. (2014). Small but powerful, the primary endosymbiont of moss bugs, Candidatus Evansia muelleri, holds a reduced genome with large biosynthetic capabilities. Genome Biol. Evol. 6, 1875-1893. doi: 10.1093/gbe/evu149

Santos-Garcia, D., Vargas-Chavez, C., Moya, A., Latorre, A., and Silva, F. J. (2015). Genome evolution in the primary endosymbiont of whiteflies sheds light on their divergence. Genome Biol. Evol. 7, 873-888. doi: 10.1093/gbe/evv038

Sloan, D. B., and Moran, N. A. (2012). Endosymbiotic bacteria as a source of carotenoids in whiteflies. Biol. Lett. 8, 986-989. doi: 10.1098/rsbl.2012.0664

Sloan, D. B., and Moran, N. A. (2013). The evolution of genomic instability in the obligate endosymbionts of whiteflies. Genome Biol. Evol. 5, 783-793. doi: $10.1093 /$ gbe/evt044

Szklarzewicz, T., Grzywacz, B., Szwedo, J., and Michalik, A. (2015). Bacterial symbionts of the leafhopper Evacanthus interruptus (Linnaeus, 1758) (Insecta, Hemiptera, Cicadellidae: Evacanthinae). Protoplasma doi: 10.1007/s00709-0150817-2 [Epub ahead of print].

Thomas, J. A., Welch, J. J., Lanfear, R., and Bromham, L. (2010). A generation time effect on the rate of molecular evolution in invertebrates. Mol. Biol. Evol. 27, 1173-1180. doi: 10.1093/molbev/msq009

Weller, C., and Wu, M. (2015). A generation-time effect on the rate of molecular evolution in bacteria. Evolution 69, 643-652. doi: 10.1111/evo.12597

Wernegreen, J. J. (2015). Endosymbiont evolution: predictions from theory and surprises from genomes. Ann. N. Y. Acad. Sci. doi: 10.1111/nyas.12740 [Epub ahead of print].

Williams, L. E., and Wernegreen, J. J. (2015). Genome evolution in an ancient bacteria-ant symbiosis: parallel gene loss among Blochmannia spanning the origin of the ant tribe Camponotini. PeerJ 3, e881. doi: 10.7717/peerj.881

Woolfit, M., and Bromham, L. (2003). Increased rates of sequence evolution in endosymbiotic bacteria and fungi with small effective population sizes. Mol. Biol. Evol. 20, 1545-1555. doi: 10.1093/molbev/msg167

Yang, Z. (2007). PAML 4: phylogenetic analysis by maximum likelihood. Mol. Biol. Evol. 24, 1586-1591. doi: 10.1093/molbev/msm088

Conflict of Interest Statement: The authors declare that the research was conducted in the absence of any commercial or financial relationships that could be construed as a potential conflict of interest.

Copyright (c) 2015 Silva and Santos-Garcia. This is an open-access article distributed under the terms of the Creative Commons Attribution License (CC BY). The use, distribution or reproduction in other forums is permitted, provided the original author(s) or licensor are credited and that the original publication in this journal is cited, in accordance with accepted academic practice. No use, distribution or reproduction is permitted which does not comply with these terms. 